\title{
Compact, integrated dynamic holographic memory with refreshed holograms
}

\author{
Jean-Jacques P. Drolet, Ernest Chuang, George Barbastathis, and Demetri Psaltis \\ Department of Electrical Engineering, Mail Stop 136-93, California Institute of Technology, Pasadena, California 91125
}

Received November 8, 1996

\begin{abstract}
An innovative architecture for compact, integrated volume holographic memories is described. It is based on phase-conjugate readout and on a modulator-detector-memory array implemented in a silicon integrated circuit. The lensless memory module sustains dynamic read-write holograms by periodic refreshing. The integrated circuit is described and experimentally characterized. Holograms were stored in a prototype storage module that uses a $30^{\circ}$-cut $\mathrm{BaTiO}_{3}$ crystal and the $90^{\circ}$ recording geometry. As many as three angularly multiplexed holograms were periodically refreshed and subjected to $>40 \%$ decay from exposure to the reference beam over 50 to 100 cycles. Experimental data are presented. (C) 1997 Optical Society of America
\end{abstract}

The use of counterpropagating reference beams for recording and readout in volume holographic memories permits compact storage module ${ }^{1-3}$ with lensless signal-beam paths. The reference beam for readout is the conjugate of the reference beam for recording. We refer to this technique as conjugate readout. Figure 1 shows a compact module that uses angular multiplexing. The module is composed of a photorefractive crystal in which holograms are stored, a pair of liquidcrystal beam steerers (one of which is hidden behind the crystal) that is responsible for angularly multiplexing holograms in the crystal, and an optoelectronic integrated circuit (OEIC) that merges the functions of a reflective spatial light modulator (SLM) for recording holograms and a detector array for readout. One writes holograms by letting the signal beam modulated by the OEIC interfere with the plane wave coming from the back of the crystal. Holograms are read out by illumination of the crystal from the front with a counterpropagating plane wave, which is the conjugate of the reference wave used to write holograms. The resulting conjugate reconstruction self-focuses back onto the OEIC. The conjugate reference can be produced by a phase-conjugate mirror, ${ }^{2}$ or it can simply consist of a counterpropagating beam in the case of plane waves. ${ }^{1,3}$

The compact module illustrated in Fig. 1 offers several benefits compared with conventional readwrite holographic systems, which use separate SLM and detector-array devices as well as imaging optics between the SLM and the detector array. Conjugate readout eliminates the reconstruction part of the signal arm and also eliminates the need for imaging optics, since it undoes the diffraction incurred by the signal beam on its way from the SLM to the recording medium. The module gains in compactness since no imaging condition imposes a spacing requirement on its elements; the module's components can be placed side by side. Conventional high-capacity memory architectures require lenses with a very high spacebandwidth product; hence, by doing away with lenses, phase-conjugate readout helps reduce system costs. Moreover, linear phase distortions are corrected by the readout process.

0146-9592/97/080552-03\$10.00/0
The OEIC enables the module shown in Fig. 1 to sustain dynamic holograms in a read-write system and simplifies the system's integration and alignment. When a proper conjugate reference is incident upon the recording material, the reconstructed signal beam is aligned at unit magnification with the photodetectors that sense it, because of the conjugate nature of the readout process and because the detectors are located within the same OEIC pixels as the modulators used to record the holograms. Furthermore, the OEIC provides a solution to the volatility of holograms stored in a read-write photorefractive memory. In such a memory, holograms slowly decay because of their exposure to the reference beam during any memory access (and also because of dark decay). The solution is to read out and reinforce the holograms periodically. ${ }^{4}$ Our approach is to sense each hologram, store it in a temporary memory, and rerecord it at the same location. This process is analogous to the wellknown refresh cycles in dynamic random-access memory. Since each pixel of the OEIC shown in Fig. 1 has memory and optical detection and modulation func-

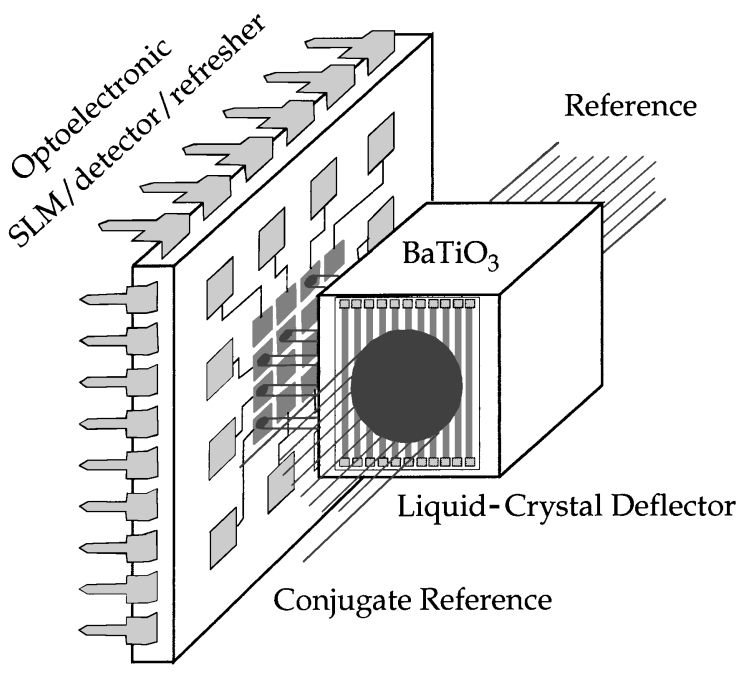

Fig. 1. Compact angularly multiplexed holographic storage module.

(C) 1997 Optical Society of America 


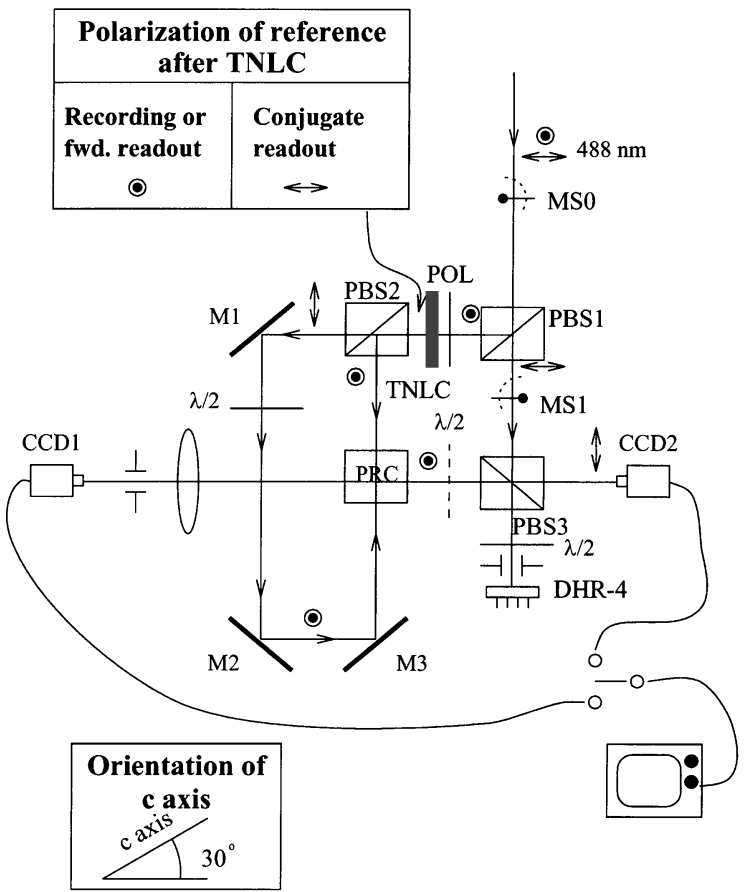

Fig. 2. Experimental setup: POL, polarizer. See text for other definitions.

tions, holograms can be refreshed in the following straightforward fashion: (1) each hologram is read out by use of the conjugate reference and internally memorized in the OEIC; (2) the memory bit within each pixel drives the corresponding modulator while the signal and the main reference beams are on, thereby reinforcing the hologram. Note that the hologramrefreshing process involves local data transfers within the pixels (detectors to memory, memory to modulators) but no transfers to or from a controlling computer. This type of OEIC is called a dynamic hologram refresher (DHR). We designed and tested a DHR chip. The silicon dies were fabricated in the Orbit Semiconductor $2.0-\mu \mathrm{m}$ double-metal $n$-well complementary metal-oxide-semiconductor process, available through MOSIS. Hybrid-aligned nematics on very large-scale integrated liquid-crystal modulators ${ }^{5}$ were fabricated in house on the devices delivered by MOSIS. The $5-\mu \mathrm{m}$ cell gap was filled with Merck E7 liquid crystal. The mean contrast was found to be 18:1. This DHR device contains an array of $20 \times 24$ pixels and is designed to appear as a static random-access memory to a controlling microprocessor. The photodetector in each pixel is a PNP active-well-substrate structure. The pixel size of this prototype is $132 \mu \mathrm{m} \times 211 \mu \mathrm{m}$. We believe that a more aggressive design, implemented in a state-of-the-art process, would yield a pixel area as small as $64 \mu \mathrm{m}^{2}$.

The experimental setup is shown in Fig. 2. Holograms were recorded in a $30^{\circ}$-cut $\mathrm{BaTiO}_{3}$ crystal (PRC). The collimated input beam of wavelength $488 \mathrm{~nm}$, controlled by mechanical shutter MS0, is split into a signal beam (going down) and a reference beam (going left) by polarizing beam splitter PBS1. The reference beam is steered either to the main reference path (directly to the PRC) or to the conjugate reference path (M1-M3, PRC) by the combined action of a $90^{\circ}$-twisted nematic liquid-crystal cell (TNLC) under computer control and a polarizing beam splitter, PBS2. The signal beam is modulated and reflected toward the recording crystal by the combination of polarizing beam splitter PBS3, a half-wave plate, and the DHR integrated circuit. The forward and conjugate reconstructions were observed

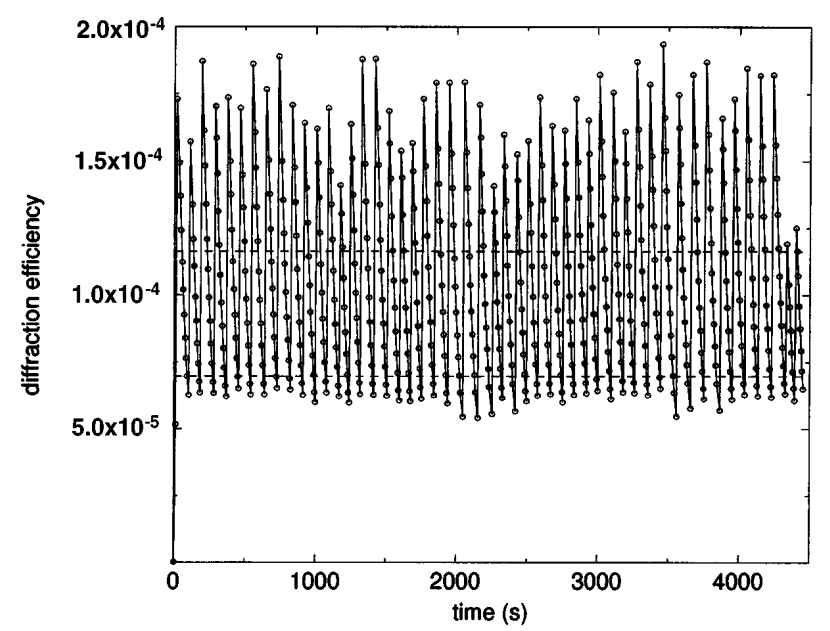

Fig. 3. Evolution of the diffraction efficiency of a hologram during 50 refresh-decay cycles.

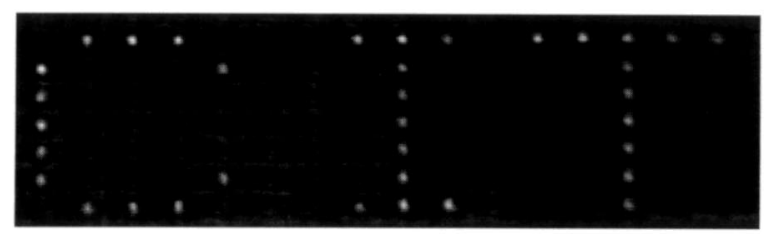

(a)

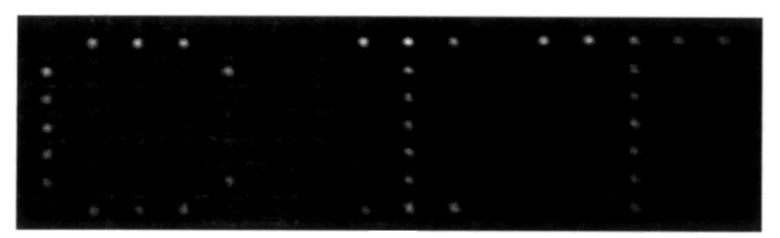

(b)

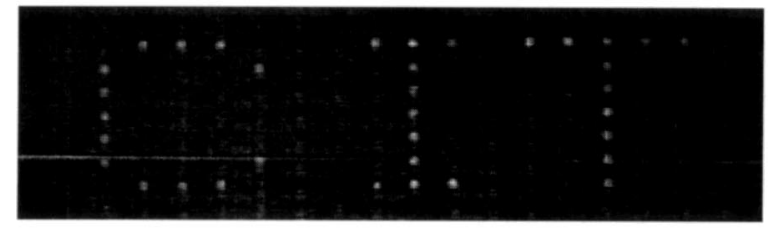

(c)

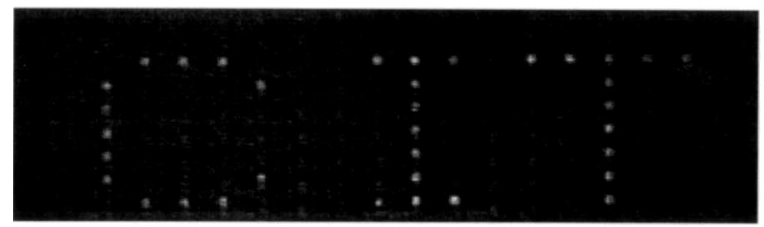

(d)

Fig. 4. (a) DHR display, (b) forward reconstruction, and conjugate reconstructions after (c) 1 and (d) 50 refreshdecay cycles. 
Table 1. Contrast Ratio, Signal-to-Noise Ratio, and Probability of Error for the Images in Fig. 4

\begin{tabular}{lccc}
\hline \multicolumn{1}{c}{ Image } & $\mathrm{CR}$ & $\mathrm{SNR}$ & $\mathrm{PE}$ \\
\hline DHR display & $15.2: 1$ & $3.76: 1$ & $1.1 \times 10^{-4}$ \\
Forward reconstruction & $18.8: 1$ & $2.24: 1$ & $2.2 \times 10^{-3}$ \\
Conjugate reconstruction & & & \\
$\quad$ cycle & $11.0: 1$ & $3.23: 1$ & $6.9 \times 10^{-4}$ \\
50 cycles & $11.7: 1$ & $3.03: 1$ & $1.0 \times 10^{-3}$ \\
\hline
\end{tabular}

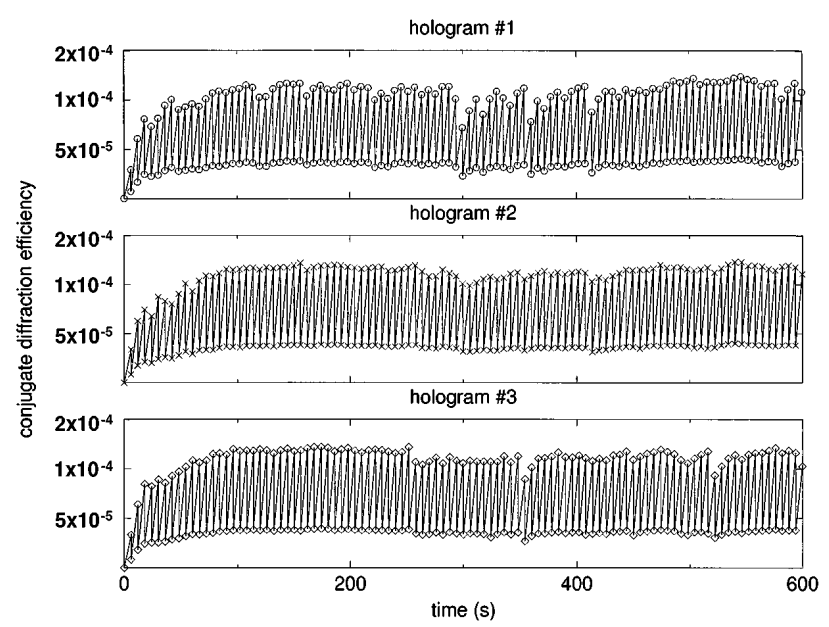

Fig. 5. Evolution of the diffraction efficiency of three multiplexed holograms during 100 refresh-decay cycles.

by use of CCD cameras, a lens, and a filtering iris; note that none of these elements is required for the normal operation of the module. A removable half-wave plate located between the recording medium and PBS3 allowed the transmission of the conjugate reconstruction through PBS3 and observation of the reconstruction with CCD camera CCD2.

A hologram of a binary pattern was recorded, allowed to decay, and refreshed for 50 cycles under computer control. Specifically, after it reached the target diffraction efficiency, $1.2 \times 10^{-4}$, the hologram was exposed to the Bragg-matched main reference until its diffraction efficiency decayed to the refresh threshold, $7.0 \times 10^{-5}$. The hologram was then reconstructed with the conjugate reference and sensed and memorized by the DHR. The signal and the main reference beams were then turned on to strengthen the hologram until the diffraction efficiency was restored to its target value. The conjugate-diffraction efficiency was $33 \%$ of the forward-diffraction efficiency. Figure 3 shows the measured conjugate-diffraction efficiency as a function of time. There were no errors at any cycle in any pixel of the detected data pages. Figure 4 shows the data page (the letters CIT), displayed on the DHR, imaged through the $\mathrm{BaTiO}_{3}$ crystal, and detected by CCD1 [Fig. 4(a)], as well as a forward reconstruction measured by CCD1 [Fig. 4(b)] and conjugate reconstructions measured by
CCD2 after 1 [Fig. 4(c)] and 50 [Fig. 4(d)] cycles. Some nonuniform attenuation is visible in the forward reconstruction [Fig. 4(b)]. It is believed to be the result of the attenuation of the reference beam as it propagates through the crystal (total transmission through the 5.7-mm-thick crystal, 12\%). However, phase-conjugate readout [Fig. 4(c)] compensates for this nonuniformity, since the reference beam enters the opposite face during readout. Table 1 lists the contrast ratio and the signal-to-noise ratio measured for each of these images, as well as the estimated probability of error. The signal-to-noise ratio is defined as $\mathrm{SNR} \equiv\left(\mu_{1}-\mu_{0}\right) /\left(\sigma_{0}^{2}+\sigma_{1}^{2}\right)^{1 / 2}$, where $\mu_{0}$ and $\mu_{1}$ are the mean values of 0 and 1 pixels, respectively, and $\sigma_{0}{ }^{2}$ and $\sigma_{1}{ }^{2}$ are the corresponding variances.

Following the original submission of this Letter, we demonstrated the periodic refreshment of three sequentially accessed angularly multiplexed holograms over 100 cycles (see Fig. 5). Multiplexing was effected by rotation of the crystal in the plane of Fig. 2 .

Although the storage module shown in Fig. 1 uses angular multiplexing, the integrative approach presented in this Letter is of a general nature. Module architectures using wavelength, shift, and phase-code multiplexing have been devised.

Note that phase-conjugate readout does not require colocated optical inputs and outputs in the pixels of the OEIC. For example, the detectors and modulators in the DHR device used in the setup shown in Fig. 2 were offset by $77 \mu \mathrm{m}$ along the direction of selectivity degeneracy (out of the figure plane). Mirror M2 was tilted vertically to introduce a small shift in the conjugate reconstruction and allow the reconstructed pixel to fall on the detectors instead of the modulators. Because of the weak out-of-plane Bragg selectivity, the small shift did not cause any appreciable attenuation or distortion of the phase-conjugate reconstruction.

This work was supported by the Advanced Research Projects Agency and the National Science Foundation Center for Neuromorphic Systems Engineering at the California Institute of Technology. J.-J. P. Drolet acknowledges support from the Natural Sciences and Engineering Research Council of Canada. The authors express their gratitude to Y. Liu for her technical assistance.

\section{References}

1. J.-J. P. Drolet, G. Barbastathis, J. S. Patel, and D. Psaltis, presented at the Optical Society of America Annual Meeting, Portland, Ore., September 10-15, 1995.

2. J.-J. P. Drolet, G. Barbastathis, and D. Psaltis, in $O p$ toelectronic Interconnects and Packaging, Vol. CR62 of SPIE Critical Reviews (Society of Photo-Optical Instrumentation Engineers, Bellingham, Wash., 1996), pp. $106-131$.

3. F. Zhao and K. Sayano, Opt. Lett. 21, 1295 (1996).

4. Y. Qiao and D. Psaltis, Opt. Lett. 17, 1376 (1992).

5. J.-J. P. Drolet, J. S. Patel, K. G. Haritos, W. Xu, A. Scherer, and D. Psaltis, Opt. Lett. 20, 2222 (1995). 